\title{
Special Issue on Molecular Robotics
}

\author{
Satoshi Murata ${ }^{1}$
}

Published online: 18 May 2020

(c) Ohmsha, Ltd. and Springer Japan KK, part of Springer Nature 2020, corrected publication 2020

Keywords Molecular robotics $\cdot$ Molecular agents $\cdot$ DNA computing $\cdot$ Molecular programming $\cdot$ Reaction diffusion systems

We have great pleasure in presenting this special issue of New Generation Computing for Molecular Robotics. This special issue includes research papers on the latest results of molecular robotics, as well as tutorial papers that introduce fundamental knowledge of molecular robotics for non-specialized readers.

One of the research papers from the forefront of DNA computing technology is on the development of an enzyme-free XOR gate [1]. In this paper, the rational design and simulation of the dynamic DNA reaction circuit are shown based on the knowledge of control engineering. Another research paper [2] is concerned with an agent model for microbial chemotaxis inspired by the behaviors of Escherichia coli and Paramecium, which gives a control strategy of molecular robots in the level of behavior.

In this special issue we have four tutorial papers. In the first tutorial [3], various computing algorithms of shape formation by many molecular robots, i.e. mobile computing entities with very weak capabilities is discussed. It gives a framework for molecular robot system in the most abstract level. The next tutorial [4] deals with implementation level design of a molecular robot system. The design, simulation, and optimization of spatio-temporal DNA computing system utilizing enzymatic reactions called "PEN Toolbox" is introduced with some illustrative examples. In the third tutorial [5], the authors review fundamental theory and computational tools for the modeling, analysis and design of stochastic biomolecular systems. The last tutorial [6] focuses on recent development of pattern generation algorithms based on DNA computing. Several typical methods to build patterns by 1-D or 2-D reaction-diffusion systems are introduced and how to implement the system by DNA molecules is explained.

Through these papers, readers can get an overview of what is being done in molecular robotics research, especially its reaction system design and behavioral control of a large number of molecular agents. We would like to express sincere

Satoshi Murata

murata@molbot.mech.tohoku.ac.jp

1 Tohoku University, Tohoku, Japan 
thanks to the authors for their contributions to this issue. We would like to add that all papers were referred in accordance with the usual rigorous standards of New Generation Computing. We gratefully acknowledge the anonymous referees for reviewing the papers in a short period of time.

Editor

Satoshi Murata

\section{References}

1. Nishijima, K., Nakakuki, T.: XOR gate design toward a practical complete set for DNA computing. New Gener Comput (2020). https://doi.org/10.1007/s00354-020-00090-3

2. Izumi, S., Azuma, S.: Chemotaxis-inspired control for multi-agent coordination: formation control by two types of chemotaxis controllers. New Gener Comput (2020). https://doi.org/10.1007/s0035 4-020-00093-0

3. Yamauchi, Y.: Distributed computing theory for molecular robot systems. New Gener Comput (2020). https://doi.org/10.1007/s00354-020-00092-1

4. Aubert-Kato, N., Cazenille, L.: Designing dynamical molecular systems with the PEN Toolbox. New Gener Comput (2020). https://doi.org/10.1007/s00354-020-00089-w

5. Hori, Y.: Modeling and analysis of stochastic reaction kinetics in biomolecular systems. New Gener Comput (2020). https://doi.org/10.1007/s00354-020-00095-y

6. Abe, K., Murata, S.: Programming methods for DNA based reaction-diffusion systems. New Gener Comput (2020). https://doi.org/10.1007/s00354-020-00094-Z

Publisher's Note Springer Nature remains neutral with regard to jurisdictional claims in published maps and institutional affiliations. 